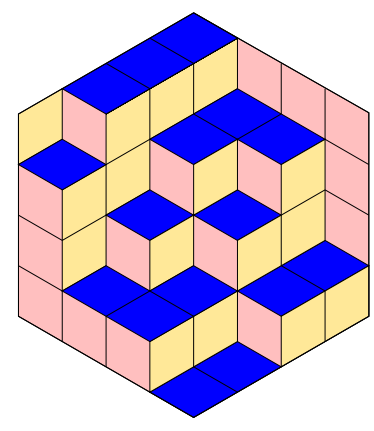

ALGEBRAIC COMBINATORICS

Eric Marberg \& Brendan Pawlowski

Principal specializations of Schubert polynomials in classical types

Volume 4, issue 2 (2021), p. 273-287.

<http://alco.centre-mersenne.org/item/ALCO_2021__4_2_273_0>

(c) The journal and the authors, 2021.

Some rights reserved.

(c) BY This article is licensed under the

Creative Commons ATtribution 4.0 International LiCEnSE.

http://creativecommons.org/licenses/by/4.0/

Access to articles published by the journal Algebraic Combinatorics on the website http://alco.centre-mersenne.org/ implies agreement with the Terms of Use (http://alco.centre-mersenne.org/legal/).

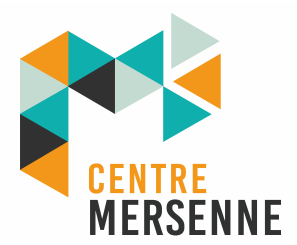

Algebraic Combinatorics is member of the Centre Mersenne for Open Scientific Publishing www.centre-mersenne.org 


\title{
Principal specializations of Schubert polynomials in classical types
}

\author{
Eric Marberg \& Brendan Pawlowski
}

\begin{abstract}
There is a remarkable formula for the principal specialization of a type A Schubert polynomial as a weighted sum over reduced words. Taking appropriate limits transforms this to an identity for the backstable Schubert polynomials recently introduced by Lam, Lee, and Shimozono. This note identifies some analogues of the latter formula for principal specializations of Schubert polynomials in classical types B, C, and D. We also describe some more general identities for Grothendieck polynomials. As a related application, we derive a simple proof of a pipe dream formula for involution Grothendieck polynomials.
\end{abstract}

\section{INTRODUCTION}

There is a remarkable formula for the principal specialization $\mathfrak{S}_{w}\left(1, q, q^{2}, \ldots, q^{n-1}\right)$ of a (type A) Schubert polynomial as a weighted sum over reduced words. Originally a conjecture of Macdonald [11], this identity was first proved algebraically by Fomin and Stanley [6]. Billey, Holroyd, and Young $[2,16]$ have recently found the first bijective proof of Macdonald's conjecture.

In this note we identify some apparently new analogues of Macdonald's identity for the principal specializations of Schubert polynomials in other classical types. Our methods are based on the algebraic techniques of Fomin and Stanley and will also lead to a simple proof of (a $K$-theoretic generalization of) the main result of [8].

To state our main theorems we need to recall a few definitions. Throughout, we let $x_{i}$ for $i \in \mathbb{Z}$ be commuting indeterminates. We use the term word to mean a finite sequence $a_{1} a_{2} \cdots a_{p}$ whose letters belong to some totally ordered alphabet. This alphabet will usually consist of the integers $\mathbb{Z}$ with their usual ordering, and in any case will always contain $(\mathbb{Z},<)$ as a subposet.

Definition 1.1. A bounded compatible sequence for a word $a=a_{1} a_{2} \cdots a_{p}$ is a weakly increasing sequence of integers $\mathbf{i}=\left(i_{1} \leqslant i_{2} \leqslant \cdots \leqslant i_{p}\right)$ with the property that

$$
i_{j}<i_{j+1} \text { whenever } a_{j} \leqslant a_{j+1} \text { and } i_{j} \leqslant a_{j} \text { whenever } 0<i_{j} .
$$

Let Compatible $(a)$ denote the set of all such sequences. Given $\mathbf{i}=\left(i_{1} \leqslant \cdots \leqslant i_{p}\right) \in$ Compatible $(a)$, define $x_{\mathbf{i}}=x_{i_{1}} \cdots x_{i_{p}}$ and write $0<\mathbf{i}$ if the numbers $i_{1}, \ldots, i_{p}$ are all positive.

\footnotetext{
Manuscript received 25th February 2020, revised 29th August 2020, accepted 31st August 2020.
}

KEYWords. Schubert polynomials, Grothendieck polynomials, Coxeter systems, reduced words.

ACKNowledgements. The first author was partially supported by Hong Kong RGC Grant ECS 26305218 . 
Let $s_{i}=(i, i+1)$ denote the permutation of $\mathbb{Z}$ interchanging $i$ and $i+1$. Fix a positive integer $n$ and let $S_{n}:=\left\langle s_{1}, s_{2}, \ldots, s_{n-1}\right\rangle \subset S_{\mathbb{Z}}:=\left\langle s_{i}: i \in \mathbb{Z}\right\rangle$. Both $S_{n}$ and $S_{\mathbb{Z}}$ are Coxeter groups with respect to their given generating sets. A reduced word for $w \in S_{\mathbb{Z}}$ is a word $a_{1} a_{2} \cdots a_{p}$ of shortest possible length such that $w=s_{a_{1}} s_{a_{2}} \cdots s_{a_{p}}$. Let $\operatorname{Reduced}(w)$ denote the set of all such words.

Definition 1.2. The Schubert polynomial of $w \in S_{n}$ is

$$
\mathfrak{S}_{w}:=\sum_{a \in \operatorname{Reduced}(w)} \sum_{0<\mathbf{i} \in \operatorname{Compatible}(a)} x_{\mathbf{i}} \in \mathbb{Z}\left[x_{1}, x_{2}, \ldots, x_{n-1}\right] .
$$

Schubert polynomials are often defined inductively using divided difference operators, following the approach of Lascoux and Schützenberger. The formula that we have given is [3, Thm. 1.1]. The identity of Macdonald [11] mentioned at the start of this introduction is as follows.

Theorem 1.3 (Fomin and Stanley [6, Thm. 2.4]). If $w \in S_{n}$ then

$$
\mathfrak{S}_{w}\left(1, q, q^{2}, \ldots, q^{n-1}\right)=\sum_{a=a_{1} a_{2} \cdots a_{p} \in \operatorname{Reduced}(w)} \frac{\left[a_{1}\right]_{q}\left[a_{2}\right]_{q} \cdots\left[a_{p}\right]_{q}}{[p]_{q} !} q^{\operatorname{comaj}(a)},
$$

where $\operatorname{comaj}(a):=\sum_{a_{i}<a_{i+1}} i$ and $[a]_{q}:=\frac{1-q^{a}}{1-q}$ and $[p]_{q} !:=[p]_{q} \cdots[2]_{q}[1]_{q}$.

Taking appropriate limits transforms the preceding formula into an identity for the backstable Schubert polynomials, which may be defined as follows.

Definition 1.4. The backstable Schubert polynomial of $w \in S_{n}$ is

$$
\overleftarrow{\mathfrak{S}}_{w}:=\sum_{a \in \operatorname{Reduced}(w)} \sum_{\mathbf{i} \in \operatorname{Compatible}(a)} x_{\mathbf{i}} \in \mathbb{Z} \llbracket \ldots, x_{-1}, x_{0}, x_{1}, \ldots, x_{n-1} \rrbracket
$$

This is the same as the formula for $\mathfrak{S}_{w}$ except now $\mathbf{i}=\left(i_{1} \leqslant i_{2} \cdots \leqslant i_{p}\right)$ may contain non-positive integers. If $w \in S_{n}$ then $\overleftarrow{\mathfrak{S}}_{w}\left(\ldots, 0,0, x_{1}, x_{2}, \ldots, x_{n-1}\right)=\mathfrak{S}_{w}$ while $\overleftarrow{\mathfrak{S}}_{w}\left(\ldots, x_{-2}, x_{-1}, x_{0}, 0,0, \ldots, 0\right)$ is the Stanley symmetric function of $w$ in the variables $x_{i}$ for $i \leqslant 0$ [10, Thm. 3.2].

Note that $\overleftarrow{\mathfrak{S}}_{w}$ is usually not a polynomial. These power series were introduced by Lam, Lee, and Shimozono [10] in connection with Schubert calculus on infinite flag varieties. They also arise as cohomology classes of degeneracy loci in products of flag varieties $[15]$.

If $F \in \mathbb{Z} \llbracket \ldots, x_{-1}, x_{0}, x_{1}, \ldots, x_{n-1} \rrbracket$ is homogeneous then the formal power series $F\left(x_{i} \mapsto q^{i-1}\right)$ obtained by setting $x_{i}=q^{i-1}$ for all integers $i<n$ is well-defined. The following result is easy to derive from Theorem 1.3 and is also a special case of Theorem 3.3. In this statement, for a word $a=a_{1} a_{2} \cdots a_{p}$ we write $\Sigma a:=\sum_{i=1}^{p} a_{i}$ and $\ell(a):=p$.

TheOREM 1.5. If $w \in S_{n}$ then $\overleftarrow{\mathfrak{S}}_{w}\left(x_{i} \mapsto q^{i-1}\right)=\sum_{a \in \operatorname{Reduced}(w)} \frac{q^{\Sigma a+\operatorname{comaj}(a)}}{(q-1)\left(q^{2}-1\right) \cdots\left(q^{\ell(a)}-1\right)}$ where the right hand expression is interpreted as a Laurent series in $q^{-1}$.

EXAMPLE 1.6. Setting $x_{i}=q^{i-1}$ in the definition of $\overleftarrow{\mathfrak{S}}_{w}$ gives another formula for $\overleftarrow{\mathfrak{S}}_{w}\left(x_{i} \mapsto q^{i-1}\right)$ as a sum over the reduced words for $w$. The corresponding terms in these two summations need not agree, however: for a given word $a=a_{1} a_{2} \cdots a_{p} \in$ $\operatorname{Reduced}(w)$, it can happen that

$$
\sum_{\mathbf{i} \in \text { Compatible }(a)} q^{\left(i_{1}-1\right)+\left(i_{2}-1\right)+\cdots+\left(i_{p}-1\right)} \neq \frac{q^{\Sigma a+\operatorname{comaj}(a)}}{(q-1)\left(q^{2}-1\right) \cdots\left(q^{p}-1\right)} .
$$


For example, if $w=(1,2)(3,4)$ and $a=1,3$ then $\sum_{\mathbf{i} \in \text { Compatible }(a)} q^{\left(i_{1}-1\right)+\cdots+\left(i_{p}-1\right)}$ is

$$
\sum_{1 \geqslant i_{1}<i_{2} \leqslant 3} q^{\left(i_{1}-1\right)+\left(i_{2}-1\right)} \in q^{2}+2 q+2+q^{-1} \mathbb{Z} \llbracket q^{-1} \rrbracket
$$

while $\frac{q^{\Sigma a+\operatorname{comaj}(a)}}{(q-1)\left(q^{2}-1\right) \cdots\left(q^{p}-1\right)}=\frac{q^{5}}{(q-1)\left(q^{2}-1\right)}$ expands into the Laurent series

$$
q^{5}\left(q^{-1}+q^{-2}+q^{-3}+\cdots\right)\left(q^{-2}+q^{-4}+\cdots\right) \in q^{2}+q+2+q^{-1} \mathbb{Z} \llbracket q^{-1} \rrbracket .
$$

For $w=(1,2)(3,4)$ there are only two reduced words and one has

$$
\begin{aligned}
\overleftarrow{\mathfrak{S}}_{(1,2)(3,4)}=\overleftarrow{e}_{1}^{2} & +\left(2 x_{1}+x_{2}+x_{3}\right) \overleftarrow{e}_{1}+x_{1}^{2}+x_{1} x_{2}+x_{1} x_{3} \\
=\cdots & +x_{0}^{2}+2 x_{-1} x_{1}+x_{-2} x_{2}+x_{-3} x_{3} \\
& +2 x_{0} x_{1}+x_{-1} x_{2}+x_{-2} x_{3} \\
& +x_{1}^{2}+x_{0} x_{2}+x_{-1} x_{3} \\
& +x_{1} x_{2}+x_{0} x_{3} \\
& +x_{1} x_{3}
\end{aligned}
$$

where $\overleftarrow{e}_{d}$ is the symmetric function $\sum_{i_{1}<i_{2}<\cdots<i_{d} \leqslant 0} x_{i_{1}} x_{i_{2}} \cdots x_{i_{d}}$. One computes

$$
\overleftarrow{\mathfrak{S}}_{(1,2)(3,4)}\left(x_{i} \mapsto q^{i-1}\right)=\frac{q^{4}}{(q-1)^{2}}=\cdots+7 q^{-4}+6 q^{-3}+5 q^{-2}+4 q^{-1}+3+2 q+q^{2}
$$

using either Theorem 1.5 or the formula $\overleftarrow{e}_{d}\left(q^{-1}, q^{-2}, \ldots\right)=\frac{1}{(q-1)\left(q^{2}-1\right) \cdots\left(q^{d}-1\right)}$

Our first new results are versions of the preceding theorem for Schubert polynomials in other classical types. We begin with type B/C. Given $0<i<n$, define $t_{i}=t_{-i}:=$ $(i, i+1)(-i,-i-1)$ and $t_{0}:=(-1,1)$. Define $W_{n}^{\mathrm{BC}}:=\left\langle t_{0}, t_{1}, \ldots, t_{n-1}\right\rangle$ to be the Coxeter group consisting of the permutations $w$ of $\mathbb{Z}$ with $w(i)=i$ for $|i|>n$ and $w(-i)=-w(i)$ for all $i \in \mathbb{Z}$.

A signed reduced word of type $B$ for an element $w \in W_{n}^{\mathrm{BC}}$ is a word $a_{1} a_{2} \cdots a_{p}$ with letters in the set $\{-n+1, \ldots,-1,0,1, \ldots, n-1\}$ of shortest possible length such that $w=t_{a_{1}} t_{a_{2}} \cdots t_{a_{p}}$. Let -0 denote a formal symbol distinct from 0 that satisfies $-1<-0<0<1$ and set $t_{-0}:=t_{0}$. A signed reduced word of type $C$ for $w \in W_{n}^{\mathrm{BC}}$ is a word $a_{1} a_{2} \cdots a_{p}$ with letters in $\{-n+1, \ldots,-1,-0,0,1, \ldots, n-1\}$ of shortest possible length such that $w=t_{a_{1}} t_{a_{2}} \cdots t_{a_{p}}$. Let $\operatorname{Reduced}_{B}^{ \pm}(w)$ and $\operatorname{Reduced}_{C}^{ \pm}(w)$ denote the respective sets of signed reduced words for $w$.

Definition 1.7. The type B/C Schubert polynomials of $w \in W_{n}^{\mathrm{BC}}$ are

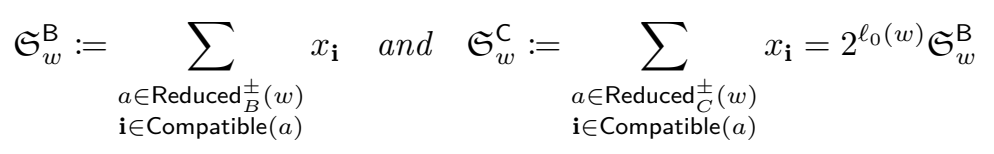

where $\ell_{0}(w):=|\{i \in \mathbb{Z}: w(i)<0<i\}|$.

Both of the "polynomials" $\mathfrak{S}_{w}^{\mathrm{B}}$ and $\mathfrak{S}_{w}^{\mathrm{C}}$ are formal power series in the ring $\mathbb{Z} \llbracket \ldots, x_{-1}, x_{0}, x_{1}, \ldots, x_{n-1} \rrbracket$. If we substitute $x_{i} \mapsto z_{i}$ for $i>0$ and $x_{i} \mapsto x_{1-i}$ for $i \leqslant 0$, then $\mathfrak{S}_{w}^{\mathrm{B}}$ and $\mathfrak{S}_{w}^{\mathrm{C}}$ specialize to the Schubert polynomials of types $\mathrm{B}$ and $\mathrm{C}$ defined by Billey and Haiman in [1]; compare our definition with [1, Thm. 3].

Let $\operatorname{Reduced}_{C}(w)$ for $w \in W_{n}^{\mathrm{BC}}$ denote the subset of words in $\operatorname{Reduced}_{C}^{ \pm}(w)$ whose letters all belong to $\{0,1, \ldots, n-1\}$. In Section 2.2 we prove the following analogue of Theorem 1.5. 
THEOREM 1.8. If $w \in W_{n}^{\mathrm{BC}}$ then

$$
\mathfrak{S}_{w}^{\mathrm{C}}\left(x_{i} \mapsto q^{i-1}\right)=\sum_{a=a_{1} a_{2} \cdots a_{p} \in \operatorname{Reduced}_{C}(w)} \frac{\left(q^{a_{1}}+1\right)\left(q^{a_{2}}+1\right) \cdots\left(q^{a_{p}}+1\right)}{(q-1)\left(q^{2}-1\right) \cdots\left(q^{p}-1\right)} q^{\operatorname{comaj}(a)}
$$

where the right hand expression is interpreted as a Laurent series in $q^{-1}$.

EXAMPLE 1.9. If $w=(1,-2)(2,-1) \in W_{n}^{\mathrm{BC}}$ then the set $\operatorname{Reduced}_{C}^{ \pm}(w)$ has 8 elements, formed by adding arbitrary signs to the letters in $a_{1} a_{2} a_{3}=0,1,0$. One can show that

$$
\begin{aligned}
\mathfrak{S}_{(1,-2)(2,-1)}^{\mathrm{C}}=4 \overleftarrow{e}_{2} & \overleftarrow{e}_{1}-4 \overleftarrow{e}_{3} \\
=\cdots & +4 x_{-2} x_{-1}^{2}+4 x_{-2}^{2} x_{0}+8 x_{-3} x_{-1} x_{0}+4 x_{-4} x_{0}^{2} \\
& +4 x_{-3} x_{0}^{2}+8 x_{-2} x_{-1} x_{0} \\
& +4 x_{-2} x_{0}^{2}+4 x_{-1}^{2} x_{0} \\
& +4 x_{-1} x_{0}^{2}
\end{aligned}
$$

where $\overleftarrow{e}_{d}:=\sum_{i_{1}<i_{2}<\cdots<i_{d} \leqslant 0} x_{i_{1}} x_{i_{2}} \cdots x_{i_{d}}$ as in Example 1.6. It follows that

$$
\begin{aligned}
\mathfrak{S}_{(1,-2)(2,-1)}^{\mathrm{C}}\left(x_{i} \mapsto q^{i-1}\right) & =\frac{4 q}{(q-1)^{2}\left(q^{3}-1\right)} \\
& =\cdots+36 q^{-9}+28 q^{-8}+20 q^{-7}+12 q^{-6}+8 q^{-5}+4 q^{-4} .
\end{aligned}
$$

We turn next to type D. For $1<i<n$, let

$$
r_{i}=r_{-i}:=(i, i+1)(-i,-i-1)=t_{i}
$$

but define

$$
r_{1}:=(1,2)(-1,-2)=t_{1} \text { and } r_{-1}:=(1,-2)(-1,2)=t_{0} t_{1} t_{0} .
$$

Define $W_{n}^{\mathrm{D}}:=\left\langle r_{-1}, r_{1}, r_{2}, \ldots, r_{n-1}\right\rangle$ to be the Coxeter group of permutations $w \in W_{n}^{\mathrm{BC}}$ for which the number of positive integers $i$ with $w(i)<0$ is even. A signed reduced word for $w \in W_{n}^{\mathrm{D}}$ is a word $a_{1} a_{2} \cdots a_{p}$ with letters in the set $\{-n+$ $1, \ldots,-2,-1,1,2, \ldots, n-1\}$ of shortest possible length such that $w=r_{a_{1}} r_{a_{2}} \cdots r_{a_{p}}$. Let $\operatorname{Reduced}_{D}^{ \pm}(w)$ denote the set of such words.

Definition 1.10. The type D Schubert polynomial of $w \in W_{n}^{\mathrm{D}}$ is

$$
\mathfrak{S}_{w}^{\mathrm{D}}=\sum_{a \in \operatorname{Reduced}_{D}^{ \pm}(w)} \sum_{\mathbf{i} \in \operatorname{Compatible}(a)} x_{\mathbf{i}} \in \mathbb{Z} \llbracket \ldots, x_{-1}, x_{0}, x_{1}, \ldots, x_{n-1} \rrbracket .
$$

If we again substitute $x_{i} \mapsto z_{i}$ for $i>0$ and $x_{i} \mapsto x_{1-i}$ for $i \leqslant 0$, then our definition of the power series $\mathfrak{S}_{w}^{\mathrm{D}}$ specializes to Billey and Haiman's formula for the Schubert polynomial of type $\mathrm{D}$ given in [1, Thm. 4].

Suppose $a=a_{1} a_{2} \cdots a_{p}$ is a sequence with $a_{i} \in\{ \pm 1, \pm 2, \pm 3, \ldots, \pm(n-1)\}$. Define

$$
\operatorname{comaj}_{\mathrm{D}}(a):=\left|\left\{i: a_{i}>0\right\}\right|+\sum_{a_{i} \prec a_{i+1}} 2 i
$$

where $\prec$ is the order $-1 \prec-2 \prec \cdots \prec-n \prec 1 \prec 2 \prec \cdots \prec n$. For example, if $a=a_{1} a_{2} a_{3} a_{4}=-1,-2,3,1$ then $\operatorname{comaj}_{\mathrm{D}}(a)=2+(2+4)=8$. We prove the following in Section 2.3.

THEOREM 1.11. If $w \in W_{n}^{\mathrm{D}}$ then

$$
\mathfrak{S}_{w}^{\mathrm{D}}\left(x_{i} \mapsto q^{i-1}\right)=\sum_{a=a_{1} a_{2} \cdots a_{p} \in \operatorname{Reduced}_{D}^{ \pm}(w)} \frac{\left(q^{\left|a_{1}\right|+1}\right)\left(q^{\left|a_{2}\right|}+1\right) \cdots\left(q^{\left|a_{p}\right|}+1\right)}{\left(q^{2}-1\right)\left(q^{4}-1\right) \cdots\left(q^{2 p}-1\right)} q^{\operatorname{comaj}_{\mathrm{D}}(a)}
$$

where the right hand expression is interpreted as a Laurent series in $q^{-1}$. 
EXAMPLE 1.12. If $w=(1,-1)(4,-4) \in W_{n}^{\mathrm{D}}$ then the set $\operatorname{Reduced}_{D}^{ \pm}(w)$ has 32 elements, formed by adding signs to the letters in $a_{1} a_{2} a_{3} a_{4} a_{5} a_{6}=3,2,1,1,2,3$ in all ways that give opposite signs to the two entries with absolute value one. One can compute that

$$
\begin{aligned}
\mathfrak{S}_{(1,-1)(4,-4)}^{\mathrm{D}}= & x_{1} x_{2} x_{3} \overleftarrow{P}_{3}+\left(x_{1} x_{2}+x_{1} x_{3}+x_{2} x_{3}\right) \overleftarrow{P}_{4}+\left(x_{1}+x_{2}+x_{3}\right) \overleftarrow{P}_{5}+\overleftarrow{P}_{6} \\
= & +x_{0}^{4} x_{1} x_{3}+2 x_{-1} x_{0}^{3} x_{2} x_{3}+2 x_{-1}^{2} x_{0} x_{1} x_{2} x_{3}+2 x_{-2} x_{0}^{2} x_{1} x_{2} x_{3} \\
& +x_{0}^{4} x_{2} x_{3}+2 x_{-1} x_{0}^{2} x_{1} x_{2} x_{3} \\
& +x_{0}^{3} x_{1} x_{2} x_{3}
\end{aligned}
$$

where $\overleftarrow{P}_{d}$ for $d>0$ is the Schur $P$-function $\frac{1}{2} \sum_{a=0}^{d} e_{a}\left(x_{0}, x_{-1}, \ldots\right) h_{d-a}\left(x_{0}, x_{-1}, \ldots\right)$ Using the formula $\overleftarrow{P}_{d}\left(q^{-1}, q^{-2}, \ldots\right)=\frac{(q+1)\left(q^{2}+1\right) \cdots\left(q^{d-1}+1\right)}{(q-1)\left(q^{2}-1\right) \cdots\left(q^{d}-1\right)}$ one can check that

$$
\begin{aligned}
\mathfrak{S}_{(1,-1)(4,-4)}^{\mathrm{D}}\left(x_{i} \mapsto q^{i-1}\right) & =\frac{q^{12}\left(q^{2}+1\right)}{(q-1)^{3}\left(q^{3}-1\right)\left(q^{5}-1\right)} \\
& =\cdots+46 q^{-5}+27 q^{-4}+15 q^{-3}+7 q^{-2}+3 q^{-1}+1,
\end{aligned}
$$

which agrees with Theorem 1.11.

Setting $q=1$ in Theorem 1.5 leads to surprising enumerative formulas involving reduced words, compatible sequences, and plane partitions [5]. By contrast, the power series $\overleftarrow{\mathfrak{S}}_{w}, \mathfrak{S}_{w}^{\mathrm{B}}, \mathfrak{S}_{w}^{\mathrm{C}}$, and $\mathfrak{S}_{w}^{\mathrm{D}}$ do not converge upon specializing $x_{i} \mapsto 1$ for all $i$. It would be interesting to find variations of our formulas with clearer enumerative content.

The second half of this note contains a few other related results. In Section 3, we extend Theorems 1.5, 1.8, and 1.11 to identities for Grothendieck polynomials. Our proofs of these formulas are fairly straightforward adaptations of the algebraic methods in $[6,9]$. It is an interesting open problem to find bijective proofs of these identities along the lines of [2].

Our approach has one other notable application, which we discuss in Section 4. There, we develop a simple alternate proof of the main result of [8], which gives a pipe dream formula for certain involution Schubert polynomials. We are able to prove a more general $K$-theoretic formula, partially resolving an open question from $[8, \S 6]$.

\section{Principal SPECializations of Schubert POlynomials}

This section contains our proofs of Theorems 1.8 and 1.11. Throughout, we fix a positive integer $n$ and let $R$ be an arbitrary commutative ring containing the ring of formal power series $\mathbb{Z} \llbracket x_{i}: i<n \rrbracket$.

2.1. Nil-Coxeter Algebras. The algebra introduced in this section figures prominently in [6] and in several of our arguments. Let $(W, S)$ be a Coxeter system with length function $\ell$. Let $\mathrm{NilCox}=\operatorname{NilCox}(W)$ be the $R$-module of all formal $R$-linear combinations of the symbols $u_{w}$ for $w \in W$. This module has a unique $R$-algebra structure with bilinear multiplication satisfying

$$
u_{v} u_{w}=\left\{\begin{array}{ll}
u_{v w} & \text { if } \ell(v w)=\ell(v)+\ell(w), \\
0 & \text { if } \ell(v w)<\ell(v)+\ell(w),
\end{array} \quad \text { for } v, w \in W\right.
$$

Following $[6, \S 2]$, we refer to NilCox as the nil-Coxeter algebra of $(W, S)$. Choose $x, y \in R$. Given $s \in S$, define $h_{s}(x):=1+x u_{s} \in$ NilCox. One checks that if $s, t \in S$ and $s t=t s$ then

$$
h_{s}(x) h_{s}(y)=h_{s}(x+y) \quad \text { and } \quad h_{s}(x) h_{t}(y)=h_{t}(y) h_{s}(x) .
$$


We will also need the following general identity, which is equivalent to [6, Lem. 5.4] after some minor changes of variables:

LEMMA 2.1 ([6, Lem. 5.4]). Let $\mathfrak{t}_{1}, \mathfrak{t}_{2}, \ldots, \mathfrak{t}_{N}$ be some elements of an R-algebra with identity 1 , and suppose $q, z_{1}, z_{2}, \ldots z_{N}$ are formal variables. Then

$$
\prod_{j=-\infty}^{0} \prod_{i=1}^{N}\left(1+q^{j-1} z_{i} \mathfrak{t}_{i}\right)=\sum_{p \geqslant 0} \sum_{a_{1}, a_{2}, \ldots, a_{p}} \frac{z_{a_{1}} z_{a_{2}} \cdots z_{a_{p}}}{(q-1)\left(q^{2}-1\right) \cdots\left(q^{p}-1\right)} q^{\operatorname{comaj}(a)} \mathfrak{t}_{a_{1}} \mathfrak{t}_{a_{2}} \cdots \mathfrak{t}_{a_{p}}
$$

where comaj $(a):=\sum_{a_{i}<a_{i+1}} i$ and the coefficients on the right are viewed as Laurent series in $q^{-1}$.

2.2. Type B/C. Here, let NilCox $=\operatorname{NilCox}\left(W_{n}^{\mathrm{BC}}\right)$ denote the nil-Coxeter algebra of type B/C Coxeter system $(W, S)=\left(W_{n}^{\mathrm{BC}},\left\{t_{0}, t_{1}, \ldots, t_{n-1}\right\}\right)$ and define $h_{i}(x):=$ $1+x u_{t_{i}} \in$ NilCox for integers $-n<i<n$ and $x \in R$. Recall that $t_{i}=t_{-i}$ so we always have $h_{i}(x)=h_{-i}(x)$. Let

$$
\begin{aligned}
A_{i}(x) & :=h_{n-1}(x) h_{n-2}(x) \cdots h_{i}(x), \\
B(x) & :=h_{n-1}(x) \cdots h_{1}(x) h_{0}(x) h_{-1}(x) \cdots h_{-n+1}(x), \\
C(x) & :=h_{n-1}(x) \cdots h_{1}(x) h_{0}(x) h_{0}(x) h_{-1}(x) \cdots h_{-n+1}(x),
\end{aligned}
$$

and note that $h_{0}(x) h_{0}(x)=h_{0}(2 x)$. Finally consider the infinite products in NilCox given by

$$
\mathfrak{S}^{\mathrm{B}}:=\prod_{i=-\infty}^{0} B\left(x_{i}\right) \prod_{i=1}^{n-1} A_{i}\left(x_{i}\right) \text { and } \mathfrak{S}^{\mathrm{C}}:=\prod_{i=-\infty}^{0} C\left(x_{i}\right) \prod_{i=1}^{n-1} A_{i}\left(x_{i}\right) .
$$

It straightforward to see that $\mathfrak{S}^{\mathrm{B}}=\sum_{w \in W_{n}^{\mathrm{BC}}} \mathfrak{S}_{w}^{\mathrm{B}} \cdot u_{w}$ and $\mathfrak{S}^{\mathrm{C}}=\sum_{w \in W_{n}^{\mathrm{BC}}} \mathfrak{S}_{w}^{\mathrm{C}} \cdot u_{w}$. Less trivially:

Proposition 2.2. It holds that

$$
\mathfrak{S}^{\mathrm{B}}=\prod_{j=-\infty}^{0}\left(h_{0}\left(x_{j}\right) \prod_{i=1}^{n-1} h_{i}\left(x_{i+j}+x_{j}\right)\right) \quad \text { and } \quad \mathfrak{S}^{\mathrm{C}}=\prod_{j=-\infty}^{0} \prod_{i=0}^{n-1} h_{i}\left(x_{i+j}+x_{j}\right) .
$$

Proof. We will just prove the formula for $\mathfrak{S}^{\mathrm{C}}$ since the other case is similar. Let

$$
\widetilde{A}_{i}(x):=h_{i}(x) h_{i+1}(x) \cdots h_{n-1}(x) .
$$

Since $A_{i}(x)=A_{i+1}(x) h_{i}(x)$ and $C(x)=A_{1}(x) h_{0}(x+x) \widetilde{A}_{1}(x)$, we have

$$
\mathfrak{S}^{\mathrm{C}}=\prod_{i=-\infty}^{-1} C\left(x_{i}\right) \cdot A_{1}\left(x_{0}\right) h_{0}\left(x_{0}+x_{0}\right) \widetilde{A}_{1}\left(x_{0}\right) A_{1}\left(x_{1}\right) A_{2}\left(x_{2}\right) \cdots A_{n-1}\left(x_{n-1}\right) .
$$

The elements $h_{i-2}(x), A_{i}(y)$, and $\widetilde{A}_{i}(z)$ all commute by [6, Lem. 4.1]. Using this fact and the identities $A_{i}(x)=A_{i+1}(x) h_{i}(x)$ and $\widetilde{A}_{i}(x)=h_{i}(x) \widetilde{A}_{i+1}(x)$, one can show

$$
h_{0}\left(x_{0}+x_{0}\right) \widetilde{A}_{1}\left(x_{0}\right) A_{1}\left(x_{1}\right) A_{2}\left(x_{2}\right) \cdots A_{n-1}\left(x_{n-1}\right)=\prod_{i=2}^{n-1} A_{i}\left(x_{i-1}\right) \cdot \prod_{i=0}^{n-1} h_{i}\left(x_{i}+x_{0}\right) .
$$

Substituting this into our formula above gives $\mathfrak{S}^{\mathrm{C}}=\mathfrak{S}^{\mathrm{C}}\left(x_{i} \mapsto x_{i-1}\right) \prod_{i=0}^{n-1} h_{i}\left(x_{i}+x_{0}\right)$ so by induction we have $\mathfrak{S}^{\mathrm{C}}=\mathfrak{S}^{\mathrm{C}}\left(x_{i} \mapsto x_{i-N}\right) \prod_{j=-N+1}^{0} \prod_{i=0}^{n-1} h_{i}\left(x_{i+j}+x_{j}\right)$ for all $N \geqslant 0$. But it is easy to see that $\lim _{N \rightarrow \infty} \mathfrak{S}^{\mathrm{C}}\left(x_{i} \mapsto x_{i-N}\right)=1$ as a limit of power series, so the result follows by sending $N \rightarrow \infty$.

We can now prove Theorem 1.8. 
Proof of Theorem 1.8. To obtain the desired formula, set $x_{i}=q^{i-1}$ in Proposition 2.2, apply Lemma 2.1 with $N=n, z_{i}=1+q^{i-1}$, and $\mathfrak{t}_{i}=u_{t_{i-1}}$, and then extract the coefficient of $u_{w}$.

2.3. Type D. Now, let NilCox $=\operatorname{Nil} \operatorname{Cox}\left(W_{n}^{\mathrm{D}}\right)$ denote the nil-Coxeter algebra of $(W, S)=\left(W_{n}^{\mathrm{D}},\left\{r_{-1}, r_{1}, \ldots, r_{n-1}\right\}\right)$ and define $h_{i}(x):=1+x u_{t_{i}} \in$ NilCox for all $i \in\{ \pm 1, \pm 2, \ldots, \pm(n-1)\}$ and $x \in R$. Let

$$
\begin{aligned}
A_{i}(x) & :=h_{n-1}(x) h_{n-2}(x) \cdots h_{i}(x), \\
\widetilde{A}_{i}(x) & :=h_{i}(x) h_{i+1}(x) \cdots h_{n-1}(x), \\
D(x) & :=h_{n-1}(x) \cdots h_{1}(x) h_{-1}(x) \cdots h_{-n+1}(x) .
\end{aligned}
$$

The Coxeter group $W_{n}^{\mathrm{D}}$ has a unique automorphism $w \mapsto w^{*}$ that maps $r_{i} \mapsto r_{-i}$ for $1 \leqslant i<n$. This map extends by linearity to an $R$-algebra automorphism of NilCox with $u_{w}^{*}:=u_{w^{*}}$. We have $A_{i}(x)^{*}=A_{i}(x)$ for $1<i<n$ and $D(x)^{*}=D(x)$, while $A_{1}(x)^{*}=h_{n-1}(x) h_{n-2}(x) \cdots h_{2}(x) h_{-1}(x)$. Consider the infinite products in NilCox

$$
\mathfrak{S}^{\mathrm{D}}:=\prod_{i=-\infty}^{0} D\left(x_{i}\right) \prod_{i=1}^{n-1} A_{i}\left(x_{i}\right) \text { and }\left(\mathfrak{S}^{\mathrm{D}}\right)^{*}:=\prod_{i=-\infty}^{0} D\left(x_{i}\right) \prod_{i=1}^{n-1} A_{i}\left(x_{i}\right)^{*} .
$$

We have $\mathfrak{S}^{\mathrm{D}}=\sum_{w \in W_{n}^{\mathrm{D}}} \mathfrak{S}_{w}^{\mathrm{D}} \cdot u_{w}$ and $\left(\mathfrak{S}^{\mathrm{D}}\right)^{*}=\sum_{w \in W_{n}^{\mathrm{D}}} \mathfrak{S}_{w}^{\mathrm{D}} \cdot u_{w^{*}}$. In addition:

Proposition 2.3. It holds that

$$
\mathfrak{S}^{\mathrm{D}}=\prod_{j=-\infty}^{0}\left(\prod_{i=1}^{n-1} h_{-i}\left(x_{i+2 j-1}+x_{2 j-1}\right) \prod_{i=1}^{n-1} h_{i}\left(x_{i+2 j}+x_{2 j}\right)\right) \text {. }
$$

Proof. Since $A_{i}(x)=A_{i+1}(x) h_{i}(x)$ and $D(x)=A_{1}(x)^{*} \widetilde{A}_{1}(x)$, we have

$$
\mathfrak{S}^{\mathrm{D}}=\prod_{i=-\infty}^{-1} D\left(x_{i}\right) \cdot A_{1}\left(x_{0}\right) * \widetilde{A}_{1}\left(x_{0}\right) A_{1}\left(x_{1}\right) A_{2}\left(x_{2}\right) \cdots A_{n-1}\left(x_{n-1}\right) .
$$

Repeating the argument in the proof of Proposition 2.2 , we deduce that $\mathfrak{S}^{\mathrm{D}}=$ $\left(\mathfrak{S}^{\mathrm{D}}\right)^{*}\left(x_{i} \mapsto x_{i-1}\right) \prod_{i=1}^{n-1} h_{i}\left(x_{i}+x_{0}\right)$. An analogous identity holds for $\left(\mathfrak{S}^{\mathrm{D}}\right)^{*}$. Alternating these formulas gives

$$
\mathfrak{S}^{\mathrm{D}}=\mathfrak{S}^{\mathrm{D}}\left(x_{i} \mapsto x_{i-2 N}\right) \prod_{j=-N+1}^{0}\left(\prod_{i=1}^{n-1} h_{-i}\left(x_{i+2 j-1}+x_{2 j-1}\right) \prod_{i=1}^{n-1} h_{i}\left(x_{i+2 j}+x_{2 j}\right)\right)
$$

for all $N \geqslant 0$. It is again easy to see that $\lim _{N \rightarrow \infty} \mathfrak{S}^{\mathrm{D}}\left(x_{i} \mapsto x_{i-2 N}\right)=1$ as a limit of formal power series, so the result follows by sending $N \rightarrow \infty$.

We can now also prove Theorem 1.11.

Proof of Theorem 1.11. By Proposition 2.3 we have

$$
\begin{aligned}
\mathfrak{S}^{\mathrm{D}}\left(x_{i}\right. & \left.\mapsto q^{i-1}\right) \\
& =\prod_{j=-\infty}^{0}\left(\prod_{i=1}^{n-1}\left(1+q^{2(j-1)} \cdot\left(1+q^{i}\right) \cdot u_{r_{-i}}\right) \cdot \prod_{i=1}^{n-1}\left(1+q^{2(j-1)} \cdot q\left(1+q^{i}\right) \cdot u_{r_{i}}\right)\right) .
\end{aligned}
$$

To get the desired expression for $\mathfrak{S}_{w}^{\mathrm{D}}$, apply Lemma 2.1 with $q$ replaced by $q^{2}$ and $N=2 n-2$ to the right side of the preceding identity, using the parameters $z_{i}=1+q^{i}$, $z_{n-1+i}=q\left(1+q^{i}\right), \mathfrak{t}_{i}=u_{r_{-i}}$, and $\mathfrak{t}_{n-1+i}=u_{r_{i}}$ for $1 \leqslant i<n$. Then extract the coefficient of $u_{w}$. 


\section{Principal specializations of Grothendieck polynomials}

In this section we describe some extensions of Theorems 1.5, 1.8, and 1.11 for Grothendieck polynomials in classical types. The identities proved here are more general but also more technical than the formulas sketched in the introduction.

3.1. ID-Coxeter algebras. Again let $(W, S)$ be an arbitrary Coxeter system with length function $\ell$. For the results in this section, we work in a generalization of the algebra NilCox $(W)$. Recall that $R$ is an arbitrary commutative ring containing $\mathbb{Z} \llbracket x_{i}$ : $i<n \rrbracket$. From this point on, we fix an element $\beta \in R$.

Let $\operatorname{IdCox}_{\beta}=\operatorname{IdCox}_{\beta}(W)$ be the $R$-module of all formal $R$-linear combinations of the symbols $\pi_{w}$ for $w \in W$. This module has a unique $R$-algebra structure with bilinear multiplication satisfying

$$
\pi_{v} \pi_{w}=\pi_{v w} \text { if } \ell(v w)=\ell(v)+\ell(w) \text { and } \pi_{s}^{2}=\beta \pi_{s}
$$

for $v, w \in W$ and $s \in S$ [9, Def. 1], which we refer to as the $i d$-Coxeter algebra of $(W, S)$. For $x, y \in R$ and $s \in S$, define

$$
x \oplus y:=x+y+\beta x y \quad \text { and } \quad h_{s}^{(\beta)}(x):=1+x \pi_{s} .
$$

Then $h_{s}^{(\beta)}(x) h_{s}^{(\beta)}(y)=h_{s}^{(\beta)}(x \oplus y)$, and if $s t=t s$ then $h_{s}^{(\beta)}(x) h_{t}^{(\beta)}(y)=h_{t}^{(\beta)}(y) h_{s}^{(\beta)}(x)$ [9, Lem. 1].

3.2. TYpe A. Let $\overleftarrow{S}_{n}:=\left\langle s_{i}: i<n\right\rangle$ be the Coxeter group of permutations $w \in S_{\mathbb{Z}}$ with $w(i)=i$ for all $i>n$. In this section we write $\operatorname{ldCox}_{\beta}=\operatorname{IdCox} \beta\left(\overleftarrow{S}_{n}\right)$ and set $\pi_{i}:=\pi_{s_{i}} \in \operatorname{IdCox}_{\beta}$ for integers $i<n$. Define $\operatorname{Hecke}(w)$ for $w \in \overleftarrow{S}_{n}$ to be the set of words $a_{1} a_{2} \cdots a_{N}$ such that $\pi_{w}=\beta^{N-\ell(w)} \pi_{a_{1}} \pi_{a_{2}} \cdots \pi_{a_{N}}$. Recall the set Compatible $(a)$ from Definition 1.1.

Definition 3.1. The backstable Grothendieck polynomial of $w \in S_{n} \subsetneq \overleftarrow{S}_{n}$ is

$$
\overleftarrow{\mathfrak{G}}_{w}:=\sum_{a \in \operatorname{Hecke}(w)} \sum_{\mathbf{i} \in \text { Compatible }(a)} \beta^{\ell(\mathbf{i})-\ell(w)} x_{\mathbf{i}} \in \mathbb{Z}[\beta] \llbracket \ldots, x_{-1}, x_{0}, x_{1}, \ldots, x_{n-1} \rrbracket .
$$

The function $\mathfrak{G}_{w}:=\overleftarrow{\mathfrak{G}}_{w}\left(\ldots, 0,0, x_{1}, x_{2}, \ldots, x_{n-1}\right)$ is the ordinary Grothendieck polynomial of $w \in S_{n}$. The power series $G_{w}:={\overleftarrow{\mathfrak{G}_{w}}}_{w}\left(\ldots, x_{3}, x_{2}, x_{1}, 0,0, \ldots, 0\right)$ given by setting $x_{i} \mapsto 0$ for $i>0$ and $x_{i} \mapsto x_{1-i}$ for $i \leqslant 0$ is a symmetric function in the $x_{i}$ variables, which is usually called the stable Grothendieck polynomial of $w \in S_{n}$.

Specializing $\beta \mapsto 0$ transforms $\overleftarrow{\mathfrak{G}}_{w} \mapsto \overleftarrow{\mathfrak{S}}_{w}$ from Section 1. The Grothendieck polynomials $\mathfrak{G}_{w}$ are closely related to the $K$-theory of flag varieties and Grassmannians $[4,13]$. We do not know of a similar geometric interpretation for the backstable Grothendieck polynomials $\overleftarrow{\mathfrak{G}}_{w}$.

For $i<n$ and $x \in R$, let

$$
h_{i}^{(\beta)}(x):=1+x \pi_{i} \quad \text { and } \quad A_{i}^{(\beta)}(x):=h_{n-1}^{(\beta)}(x) h_{n-2}^{(\beta)}(x) \cdots h_{i}^{(\beta)}(x) .
$$

Define

$$
\overleftarrow{\mathfrak{G}}:=\cdots A_{n-3}^{(\beta)}\left(x_{n-3}\right) A_{n-2}^{(\beta)}\left(x_{n-2}\right) A_{n-1}^{(\beta)}\left(x_{n-1}\right)=\prod_{i=-\infty}^{n-1} A_{i}^{(\beta)}\left(x_{i}\right) \in \mathrm{IdCox}_{\beta}
$$

If $w \in S_{n}$ then the coefficient of $\pi_{w}$ in this expression is $\overleftarrow{\mathfrak{G}}_{w}$.

Proposition 3.2. It holds that $\overleftarrow{\mathfrak{G}}=\prod_{j=-\infty}^{0} \prod_{i=-\infty}^{n-1} h_{i}^{(\beta)}\left(x_{i+j}\right)$ 
Proof. We have $\overleftarrow{\mathfrak{G}}=\cdots A_{1}^{(\beta)}\left(x_{0}\right) h_{0}^{(\beta)}\left(x_{0}\right) A_{2}^{(\beta)}\left(x_{1}\right) h_{1}^{(\beta)}\left(x_{1}\right) \cdots A_{n-1}^{(\beta)}\left(x_{n-2}\right) h_{n-1}^{(\beta)}\left(x_{n-1}\right)$ by definition. As $h_{i}^{(\beta)}(x)$ and $A_{i+2}^{(\beta)}(y)$ commute, it follows that $\overleftarrow{\mathfrak{G}}=\overleftarrow{\mathfrak{G}}\left(x_{i} \mapsto\right.$ $\left.x_{i-1}\right) \prod_{i=-\infty}^{n-1} h_{i}^{(\beta)}\left(x_{i}\right)$ so by induction $\overleftarrow{\mathfrak{G}}=\overleftarrow{\mathfrak{G}}\left(x_{i} \mapsto x_{i-N}\right) \prod_{j=-N+1}^{0} \prod_{i=-\infty}^{n-1} h_{i}^{(\beta)}\left(x_{i+j}\right)$ for all $N \geqslant 0$. But we have $\lim _{N \rightarrow \infty} \overleftarrow{\mathfrak{G}}\left(x_{i} \mapsto x_{i-N}\right)=1$ as a limit of formal power series, so the result follows by sending $N \rightarrow \infty$.

We can now prove the following generalization of Theorem 1.5.

Theorem 3.3. If $w \in S_{n} \subsetneq \overleftarrow{S}_{n}$ then

$$
\overleftarrow{\mathfrak{G}}_{w}\left(x_{i} \mapsto q^{i-1}\right)=\sum_{a \in \operatorname{Hecke}(w)} \frac{\beta^{\ell(a)-\ell(w)}}{(q-1)\left(q^{2}-1\right) \cdots\left(q^{\ell(a)}-1\right)} q^{\Sigma a+\operatorname{comaj}(a)}
$$

where the right hand expression is interpreted as a Laurent series in $q^{-1}$.

Proof. If $w \in S_{n}$ then the coefficient of $\pi_{w}$ in $\overleftarrow{\mathfrak{G}}$ is the same as the coefficient of $\pi_{w}$ in the truncated product $\prod_{j=-\infty}^{0} \prod_{i=1}^{n-1} h_{i}^{(\beta)}\left(x_{i+j}\right)$. This coefficient is $\overleftarrow{\mathfrak{G}}_{w}$, and the theorem follows by applying Lemma 2.1 with $N=n-1$ and $z_{i} \mathfrak{t}_{i}=q^{i} \pi_{s_{i}}$ to the latter expression.

There are Grothendieck polynomials in the other classical types [9] which generalize $\mathfrak{S}_{w}^{\mathrm{B}}, \mathfrak{S}_{w}^{\mathrm{C}}$, and $\mathfrak{S}_{w}^{\mathrm{D}}$ in the same way that $\overleftarrow{\mathfrak{G}}_{w}$ generalizes $\overleftarrow{\mathfrak{S}}_{w}$. We discuss these formal power series next.

3.3. Type B/C. In this section let $\operatorname{IdCox}_{\beta}=\operatorname{IdCox}_{\beta}\left(W_{n}^{\mathrm{BC}}\right)$ and write $\pi_{i}:=\pi_{t_{i}} \in$ $\operatorname{IdCox}_{\beta}$ for $-n<i<n$. Given a permutation $w \in W_{n}^{\mathrm{BC}}$, define $\operatorname{Hecke}_{B}^{ \pm}(w)$ and $\operatorname{Hecke}_{C}^{ \pm}(w)$ to be the sets of words $a_{1} a_{2} \cdots a_{N}$, with letters in $\{-n+$ $1, \ldots,-1,0,1, \ldots, n-1\}$ and $\{-n+1<\cdots<-1<-0<0<1<\cdots<n-1\}$, respectively, such that $\pi_{w}=\beta^{N-\ell(w)} \pi_{a_{1}} \pi_{a_{2}} \cdots \pi_{a_{N}} \in \mathrm{IdCox}_{\beta}$, where $\ell(w)$ denotes the usual Coxeter length of $w$ and $\pi_{-0}:=\pi_{0} \in \operatorname{IdCox}_{\beta}$. Recall that we view -0 as a symbol distinct from 0 .

Definition 3.4. The type $\mathrm{B} / \mathrm{C}$ Grothendieck polynomials of $w \in W_{n}^{\mathrm{BC}}$ are

$$
\mathfrak{G}_{w}^{\mathrm{B}}:=\sum_{\substack{a \in \operatorname{Hecke} \\ \mathbf{i} \in \operatorname{Compatible}(a)}} \beta^{\ell(\mathbf{i})-\ell(w)} x_{\mathbf{i}} \text { and } \mathfrak{G}_{w}^{\mathrm{C}}:=\sum_{\substack{a \in \operatorname{Hecke} e_{C}^{ \pm}(w) \\ \mathbf{i} \in \operatorname{Compatible}(a)}} \beta^{\ell(\mathbf{i})-\ell(w)} x_{\mathbf{i}} .
$$

We may consider the finite sums

$$
\mathfrak{G}^{\mathrm{B}}:=\sum_{w \in W_{n}^{\mathrm{BC}}} \mathfrak{G}_{w}^{\mathrm{B}} \cdot \pi_{w} \in \operatorname{IdCox} \beta\left(W_{n}^{\mathrm{BC}}\right) \quad \text { and } \quad \mathfrak{G}^{\mathrm{C}}:=\sum_{w \in W_{n}^{\mathrm{BC}}} \mathfrak{G}_{w}^{\mathrm{C}} \cdot \pi_{w} \in \operatorname{IdCox} \beta\left(W_{n}^{\mathrm{BC}}\right) .
$$

Define $A_{i}^{(\beta)}(x), B^{(\beta)}(x)$, and $C^{(\beta)}(x)$ as in (2) but with $h_{i}(x)$ replaced by

$$
h_{i}^{(\beta)}(x):=1+x \pi_{i} \in \operatorname{Id} \operatorname{Cox}_{\beta}\left(W_{n}^{\mathrm{BC}}\right) \quad \text { for }-n<i<n \text { and } x \in R .
$$

Then $\mathfrak{G}^{\mathrm{B}}$ and $\mathfrak{G}^{\mathrm{C}}$ are given by the formulas in (3) with $A_{i}, B, C$ replaced by $A_{i}^{(\beta)}$, $B^{(\beta)}, C^{(\beta)}$. Comparing with [9, Def. 9] shows that $\mathfrak{G}_{w}^{\mathrm{B}}$ and $\mathfrak{G}_{w}^{\mathrm{C}}$ are obtained from Kirillov and Naruse's double Grothendieck polynomials $\mathcal{G}_{w}^{\mathrm{B}}(a, b ; x)$ and $\mathcal{G}_{w}^{\mathrm{C}}(a, b ; x)$ by setting $a_{i} \mapsto x_{i}, b_{i} \mapsto 0$, and $x_{i} \mapsto x_{1-i}$.

Proposition 3.5. It holds that

$$
\mathfrak{G}^{\mathrm{B}}=\prod_{j=-\infty}^{0}\left(h_{0}^{(\beta)}\left(x_{j}\right) \prod_{i=1}^{n-1} h_{i}^{(\beta)}\left(x_{i+j} \oplus x_{j}\right)\right) \quad \text { and } \quad \mathfrak{G}^{\mathrm{C}}=\prod_{j=-\infty}^{0} \prod_{i=0}^{n-1} h_{i}^{(\beta)}\left(x_{i+j} \oplus x_{j}\right) .
$$


Proof. Since $A_{i}^{(\beta)}(x)$ commutes with $\widetilde{A}_{i}^{(\beta)}(x):=h_{i}^{(\beta)}(x) h_{i+1}^{(\beta)}(x) \cdots h_{n-1}^{(\beta)}(x)$ by $[9$, Lem. 3], the result follows by the same proof as Proposition 2.2, mutatis mutandis.

Given a word $a=a_{1} a_{2} \cdots a_{p}$ with $a_{i} \in\{-n+1<\cdots<-1<-0<0<1<\cdots<$ $n-1\}$, let $I(a)$ be the set of indices $i \in[p]$ with $a_{i} \in\{1,2, \ldots, n-1\}$ and define

$$
\Sigma_{\mathrm{BC}}(a):=\sum_{i \in I(a)} a_{i} \text { and } \operatorname{comaj}_{\mathrm{BC}}(a):=\sum_{a_{i} \prec a_{i+1}} i
$$

where $\prec$ is the order $-0 \prec 0 \prec-1 \prec 1 \prec-2 \prec 2 \prec \ldots$ For example, if $a=$ $-1,1,-2,1$ then $\Sigma_{\mathrm{BC}}(a)=1+1=2$ and $\operatorname{comaj}_{\mathrm{BC}}(a)=1+2=3$.

THEOREM 3.6. If $w \in W_{n}^{\mathrm{BC}}$ then the following identities hold:

(1) $\mathfrak{G}_{w}^{\mathrm{B}}\left(x_{i} \mapsto q^{i-1}\right)=\sum_{a \in \operatorname{Hecke}_{B}^{ \pm}(w)} \frac{\beta^{\ell(a)-\ell(w)}}{(q-1)\left(q^{2}-1\right) \cdots\left(q^{\ell(a)}-1\right)} q^{\Sigma_{\mathrm{BC}}(a)+\operatorname{comaj}_{\mathrm{BC}}(a)}$

(2) $\mathfrak{G}_{w}^{\mathrm{C}}\left(x_{i} \mapsto q^{i-1}\right)=\sum_{a \in \operatorname{Hecke}_{C}^{ \pm}(w)} \frac{\beta^{\ell(a)-\ell(w)}}{(q-1)\left(q^{2}-1\right) \cdots\left(q^{\ell(a)}-1\right)} q^{\Sigma_{\mathrm{BC}}(a)+\operatorname{comaj}_{\mathrm{BC}}(a)}$.

The right hand expressions in both parts are interpreted as Laurent series in $q^{-1}$.

The second identity reduces to Theorem 1.8 when $\beta=0$ since the sum $\sum_{a} q^{\Sigma_{\mathrm{BC}}(a)+\operatorname{comaj}_{\mathrm{BC}}(a)}$ over all words $a=a_{1} a_{2} \cdots a_{p} \in \operatorname{Reduced}_{C}^{ \pm}(w)$ with the same unsigned form is exactly the product $\left(q^{\left|a_{1}\right|}+1\right)\left(q^{\left|a_{2}\right|}+1\right) \cdots\left(q^{\left|a_{p}\right|}+1\right) q^{\operatorname{comaj}\left(\left|a_{1}\right|\left|a_{2}\right| \cdots\left|a_{p}\right|\right)}$.

Proof. Part (1) is similar so we just prove (2). As $h_{i}^{(\beta)}\left(x_{i+j} \oplus x_{j}\right)=h_{i}^{(\beta)}\left(x_{j}\right) h_{i}^{(\beta)}\left(x_{i+j}\right)$, we have

$$
\mathfrak{G}^{\mathrm{C}}\left(x_{i} \mapsto q^{i-1}\right)=\prod_{j=-\infty}^{0} \prod_{i=0}^{n-1}\left(1+q^{j-1} \cdot \pi_{i}\right)\left(1+q^{j-1} \cdot q^{i} \cdot \pi_{i}\right)
$$

by Proposition 3.5. The identity for $\mathfrak{G}_{w}^{\mathrm{C}}$ follows by extracting the coefficient of $\pi_{w}$ from the right side after applying Lemma 2.1 with $N=2 n$ and with the parameters $z_{1}, z_{2}, \ldots, z_{2 n}$ and $\mathfrak{t}_{1}, \mathfrak{t}_{2}, \ldots, \mathfrak{t}_{2 n}$ replaced by $1,1,1, q, 1, q^{2}, \ldots, 1, q^{n-1}$ and $\pi_{0}, \pi_{0}, \pi_{1}, \pi_{1}, \ldots, \pi_{n-1}, \pi_{n-1}$, respectively.

3.4. TyPE D. In this section let $\operatorname{IdCox}_{\beta}=\operatorname{IdCox}_{\beta}\left(W_{n}^{\mathrm{D}}\right)$ and $\pi_{i}:=\pi_{r_{i}} \in \operatorname{IdCox}_{\beta}$. Given $w \in W_{n}^{\mathrm{D}}$, let $\operatorname{Hecke}_{D}^{ \pm}(w)$ be the set of words $a_{1} a_{2} \cdots a_{N}$ with letters in $[ \pm(n-1)]:=$ $\{ \pm 1, \pm 2, \ldots, \pm(n-1)\}$ such that $\pi_{w}=\beta^{N-\ell(w)} \pi_{a_{1}} \pi_{a_{2}} \cdots \pi_{a_{N}} \in \mathrm{IdCox}_{\beta}$, where $\ell(w)$ is the usual Coxeter length.

Definition 3.7. The type D Grothendieck polynomial of $w \in W_{n}^{\mathrm{D}}$ is

$$
\mathfrak{G}_{w}^{\mathrm{D}}:=\sum_{a \in \operatorname{Hecke}_{D}^{ \pm}(w)} \sum_{\mathbf{i} \in \operatorname{Compatible}(a)} \beta^{\ell(\mathbf{i})-\ell(w)} x_{\mathbf{i}} .
$$

We consider the sum

$$
\mathfrak{G}^{\mathrm{D}}:=\sum_{w \in W_{n}^{\mathrm{D}}} \mathfrak{G}_{w}^{\mathrm{D}} \cdot \pi_{w} \in \operatorname{IdCox}_{\beta}\left(W_{n}^{\mathrm{D}}\right) .
$$

If we define $A_{i}^{(\beta)}(x)$ and $D^{(\beta)}(x)$ as in (4) but with $h_{i}(x)$ replaced by

$$
h_{i}^{(\beta)}(x):=1+x \pi_{i} \in \operatorname{IdCox}_{\beta}\left(W_{n}^{\mathrm{D}}\right) \quad \text { for } i \in[ \pm(n-1)] \text { and } x \in R,
$$

then $\mathfrak{G}^{\mathrm{D}}$ is given by the formula in (5) with $A_{i}$ and $D$ replaced by $A_{i}^{(\beta)}$ and $D^{(\beta)}$. Comparing with [9, Def. 9] shows that $\mathfrak{G}_{w}^{\mathrm{D}}$ is obtained from Kirillov and Naruse's double Grothendieck polynomial $\mathcal{G}_{w}^{\mathrm{D}}(a, b ; x)$ by making the substitutions $a_{i} \mapsto x_{i}$, $b_{i} \mapsto 0$, and $x_{i} \mapsto x_{1-i}$. 
Proposition 3.8. It holds that

$$
\mathfrak{G}^{\mathrm{D}}=\prod_{j=-\infty}^{0}\left(\prod_{i=1}^{n-1} h_{-i}^{(\beta)}\left(x_{i+2 j-1} \oplus x_{2 j-1}\right) \prod_{i=1}^{n-1} h_{i}^{(\beta)}\left(x_{i+2 j} \oplus x_{2 j}\right)\right) .
$$

Proof. Similar to Proposition 3.5, the result follows by repeating the proof of Proposition 2.3 after adding " $(\beta)$ " superscripts to all relevant symbols and substituting $h_{i}^{(\beta)}(x) h_{i}^{(\beta)}(y)=h_{i}^{(\beta)}(x \oplus y)$ wherever the identity $h_{i}(x) h_{i}(y)=h_{i}(x+y)$ is used.

To state an analogue of Theorem 1.11 for $\mathfrak{G}_{w}^{\mathrm{D}}$, we consider the ordered alphabet

$$
\left\{-1^{\prime} \prec-1 \prec-2^{\prime} \prec-2 \prec \cdots \prec-n^{\prime} \prec-n \prec 1^{\prime} \prec 1 \prec 2^{\prime} \prec 2 \prec \cdots \prec n^{\prime} \prec n\right\} .
$$

If $w \in W_{n}^{\mathrm{D}}$ then let PrimedHecke ${ }_{D}^{ \pm}(w)$ denote the set of words in this alphabet which become elements of $\operatorname{Hecke}_{D}^{ \pm}(w)$ when all primes are removed from its letters. Given such a word $a=a_{1} a_{2} \cdots a_{p}$, let $J(a)$ be the set of indices $i \in[p]$ for which $a_{i}$ is unprimed, and define

$$
\Sigma_{\mathrm{D}}(a):=\sum_{i \in J(a)}\left|a_{i}\right| \text { and } \operatorname{comaj}_{\mathrm{D}}(a):=\left|\left\{i: a_{i} \in\left\{1^{\prime}, 1,2^{\prime}, 2, \ldots\right\}\right\}\right|+\sum_{a_{i} \prec a_{i+1}} 2 i \text {. }
$$

For example, if $a=2^{\prime},-1^{\prime},-1,-3,2$ then $\Sigma_{\mathrm{D}}(a)=1+3+2=6$ and $\operatorname{comaj}_{\mathrm{D}}(a)=$ $2+(4+6+8)=20$.

THEOREM 3.9. If $w \in W_{n}^{\mathrm{D}}$ then

$$
\mathfrak{G}_{w}^{\mathrm{D}}\left(x_{i} \mapsto q^{i-1}\right)=\sum_{a \in \text { PrimedHecke }_{D}^{ \pm}(w)} \frac{\beta^{\ell(a)-\ell(w)}}{\left(q^{2}-1\right)\left(q^{4}-1\right) \cdots\left(q^{2 \ell(a)}-1\right)} q^{\Sigma_{\mathrm{D}}(a)+\operatorname{comaj}_{\mathrm{D}}(a)}
$$

where the right hand expression is interpreted as a Laurent series in $q^{-1}$.

As with Theorem 3.6, this identity reduces to Theorem 1.11 when $\beta=0$.

Proof. The proof is similar to Theorem 3.6. Proposition 3.8 implies that $\mathfrak{G}^{\mathrm{D}}\left(x_{i} \mapsto\right.$ $\left.q^{i-1}\right)$ is

$$
\begin{aligned}
\prod_{j=-\infty}^{0}\left(\prod_{i=1}^{n-1}\left(1+q^{2(j-1)} \cdot \pi_{-i}\right)\left(1+q^{2(j-1)} \cdot q^{i} \cdot \pi_{-i}\right)\right. \\
\\
\left.\prod_{i=1}^{n-1}\left(1+q^{2(j-1)} \cdot q \cdot \pi_{i}\right)\left(1+q^{2(j-1)} \cdot q^{i+1} \cdot \pi_{i}\right)\right) .
\end{aligned}
$$

The identity for $\mathfrak{G}_{w}^{\mathrm{D}}$ follows by extracting the coefficient of $\pi_{w}$ from this expression after applying Lemma 2.1 with $q$ replaced by $q^{2}$ and with $N=4 n-4$. When applying the lemma, we set the parameters $z_{1}, z_{2}, \ldots, z_{2 n-2}$ (respectively, $\left.z_{2 n-1}, z_{2 n}, \ldots, z_{4 n-4}\right)$ to $1, q, 1, q^{2}, 1, q^{3} \ldots$ (respectively, $q, q^{2}, q, q^{3}, q, q^{4} \ldots$ ), while taking $\mathfrak{t}_{1}, \mathfrak{t}_{2}, \ldots, \mathfrak{t}_{2 n-2}\left(\right.$ respectively, $\left.\mathfrak{t}_{2 n-1}, \mathfrak{t}_{2 n}, \ldots, \mathfrak{t}_{4 n-4}\right)$ to be $\pi_{-1}, \pi_{-1}, \pi_{-2}, \pi_{-2}, \ldots$ (respectively, $\pi_{1}, \pi_{1}, \pi_{2}, \pi_{2}, \ldots$ ).

\section{Involution Grothendieck POLYNomials}

This final section is something of a digression. Here, we reuse the techniques introduced above to give a simple proof of a new formula for certain involution Grothendieck polynomials.

In this section, we let $\operatorname{IdCox}_{\beta}=\operatorname{IdCox}_{\beta}\left(S_{n}\right)$ be the id-Coxeter algebra for the finite Coxeter system $(W, S)=\left(S_{n},\left\{s_{1}, s_{2}, \ldots, s_{n-1}\right\}\right)$, and write $\pi_{i}:=\pi_{s_{i}} \in \mathrm{IdCox}_{\beta}$. Let

$$
\mathcal{I}_{n}:=\left\{w \in S_{n}: w=w^{-1}\right\} \quad \text { and } \quad \mathcal{I}_{n}^{\mathrm{FPF}}:=\left\{w^{-1} 1^{\mathrm{FPF}} w: w \in S_{n}\right\}
$$


where $1^{\mathrm{FPF}}=\cdots(1,2)(3,4)(5,6) \cdots$ denotes the permutation of $\mathbb{Z}$ mapping $i \mapsto$ $i-(-1)^{i}$. The sets $\mathcal{I}_{n}$ and $\mathcal{I}_{n}^{\text {FPF }}$ are always disjoint, although when $n$ is even the elements of $\mathcal{I}_{n}^{\mathrm{FPF}}$ are naturally in bijection with the fixed-point-free elements of $\mathcal{I}_{n}$.

Let InvolMod $\beta$ and FixedMod ${ }_{\beta}$ denote the free $R$-modules consisting of all $R$-linear combinations of the symbols $m_{z}$ for $z \in \mathcal{I}_{n}$ and $z \in \mathcal{I}_{n}^{\mathrm{FPF}}$, respectively. These sets have unique right $\mathrm{IdCox}_{\beta}$-module structures (see [12, 1.2 and $\left.\S 1.3\right]$ ) satisfying, for each integer $1 \leqslant i<n$,

$$
m_{z} \pi_{i}=\left\{\begin{array}{ll}
m_{z s_{i}} & \text { if } z(i)<z(i+1) \text { and } z s_{i}=s_{i} z, \\
m_{s_{i} z s_{i}} & \text { if } z(i)<z(i+1) \text { and } z s_{i} \neq s_{i} z, \\
\beta m_{z} & \text { if } z(i)>z(i+1),
\end{array} \quad \text { for } z \in \mathcal{I}_{n}\right.
$$

and

$$
m_{z} \pi_{i}=\left\{\begin{array}{ll}
m_{s_{i} z s_{i}} & \text { if } z(i)<z(i+1), \\
\beta m_{z} & \text { if } i+1 \neq z(i)>z(i+1) \neq i, \\
0 & \text { if } i+1=z(i)>z(i+1)=i,
\end{array} \quad \text { for } z \in \mathcal{I}_{n}^{\mathrm{FPF}} .\right.
$$

An involution Hecke word for $z \in \mathcal{I}_{n}$ is a word $a_{1} a_{2} \cdots a_{p}$ such that

$$
m_{1} \pi_{a_{1}} \pi_{a_{2}} \cdots \pi_{a_{p}}=\beta^{N} m_{z} \in \operatorname{InvolMod}_{\beta} \text { for some integer } N \geqslant 0 \text {. }
$$

To avoid excessive subscripts, define

$$
m_{1}^{\mathrm{FPF}}:=m_{1} \mathrm{FPF} \in \text { FixedMod }_{\beta} .
$$

An involution Hecke word for $z \in \mathcal{I}_{n}^{\mathrm{FPF}}$ is a word $a_{1} a_{2} \cdots a_{p}$ such that

$$
m_{1}^{\mathrm{FPF}} \pi_{a_{1}} \pi_{a_{2}} \cdots \pi_{a_{p}}=\beta^{N} m_{z} \in \text { FixedMod }_{\beta} \text { for some integer } N \geqslant 0,
$$

assuming $\beta^{N} \neq 0$ for $N \geqslant 0$. Neither of these definitions depends on $\beta$, but in the fixed-point-free case we wish to exclude words $a_{1} a_{2} \cdots a_{p}$ for which $z:=s_{a_{i-1}} \cdots s_{a_{2}} s_{a_{1}} 1^{\mathrm{FPF}} s_{a_{1}} s_{a_{2}} \ldots s_{a_{i-1}}$ has $a_{i}+1=z\left(a_{i}\right)>z\left(a_{i}+1\right)=a_{i}$ for some $i$.

Let InvHecke $(z)$ denote the set of involution Hecke words for an element $z$ in $\mathcal{I}_{n}$ or $\mathcal{I}_{n}^{\mathrm{FPF}}$. This set was denoted as either $\mathcal{H}^{\mathrm{O}}(z)$ for $z \in \mathcal{I}_{n}$ or $\mathcal{H}^{\mathrm{Sp}}(z)$ for $z \in \mathcal{I}_{n}^{\mathrm{FPF}}$ in [12]. Also define

$$
\widehat{\ell}(z)=\min \{\ell(a): a \in \operatorname{InvHecke}(z)\} .
$$

For an explicit formula for $\widehat{\ell}$, see [12, Eq. (5.1)].

EXAMPLE 4.1. If $y=s_{3} s_{2} s_{3}=s_{2} s_{3} s_{2}=(2,4) \in \mathcal{I}_{n}$, then $\operatorname{InvHecke}(y)$ is the set of all finite words on the alphabet $\{2,3\}$ in which 2 and 3 both appear. If $w=(2,3,4)=$ $s_{2} s_{3} \in S_{n}$ and $z=w^{-1} 1^{\mathrm{FPF}} w=\cdots(-3,-2)(-1,0)(1,4)(2,3)(5,6)(7,8) \cdots \in \mathcal{I}_{n}^{\mathrm{FPF}}$, then $\operatorname{InvHecke}(z)$ is the set of words obtained by prepending 2 to a nonempty word on $\{1,3\}$. In either case $\widehat{\ell}(y)=\widehat{\ell}(z)=2$.

Our final theorem concerns these analogues of $\mathfrak{G}_{w}$ :

Definition 4.2. The involution Grothendieck polynomial of $z \in \mathcal{I}_{n} \sqcup \mathcal{I}_{n}^{\mathrm{FPF}}$ is

$$
\widehat{\mathfrak{G}}_{z}:=\sum_{a \in \operatorname{InvHecke}(z)} \sum_{0<\mathbf{i} \in \operatorname{Compatible}(a)} \beta^{\ell(\mathbf{i})-\widehat{\ell}(z)} x_{\mathbf{i}} \in \mathbb{Z}[\beta]\left[x_{1}, x_{2}, \ldots, x_{n-1}\right] .
$$

If $n$ is even and $z \in \mathcal{I}_{n}^{\mathrm{FPF}}$ then $\widehat{\mathfrak{G}}_{z}$ coincides with the symplectic Grothendieck polynomials $\mathfrak{G}_{z}^{\mathrm{Sp}}$ studied in [13, 14]. The paper [13] also introduces certain orthogonal Grothendieck polynomials $\mathfrak{G}_{z}^{\mathrm{O}}$ indexed by $z \in \mathcal{I}_{n}$, but these are generally not the same as $\widehat{\mathfrak{G}}_{z}$. However, $\widehat{\mathfrak{G}}_{z}$ does specialize when $\beta=0$ to both kinds of involution Schubert polynomials $\widehat{\mathfrak{S}}_{z}$ and $\widehat{\mathfrak{S}}_{z}^{\mathrm{FPF}}$ considered in $[7,8]$. 
Because InvolMod $\beta$ and FixedMod $\beta$ are $\operatorname{IdCox}_{\beta}$-modules, there exists for each $z \in$ $\mathcal{I}_{n} \sqcup \mathcal{I}_{n}^{\text {FPF }}$ a set HeckeAtoms $(z) \subset S_{n}$ (see [12, § 2.1]) such that

(9) $\operatorname{InvHecke}(z)=\bigsqcup_{w \in \operatorname{HeckeAtoms}(z)} \operatorname{Hecke}(w)$ and $\widehat{\mathfrak{G}}_{z}=\sum_{w \in \operatorname{HeckeAtoms}(z)} \beta^{\ell(w)-\widehat{\ell}(z)} \mathfrak{G}_{w}$

where

$$
\mathfrak{G}_{w}:=\overleftarrow{\mathfrak{G}}_{w}\left(\ldots, 0,0, x_{1}, x_{2}, \ldots, x_{n-1}\right)=\sum_{a \in \operatorname{Hecke}(w)} \sum_{0<\mathbf{i} \in \operatorname{Compatible}(a)} \beta^{\ell(\mathbf{i})-\ell(w)} x_{\mathbf{i}}
$$

for $w \in S_{n}$.

Again let $h_{i}^{(\beta)}(x):=1+x \pi_{i} \in \mathrm{IdCox}_{\beta}$ and define

$$
A_{i}^{(\beta)}(x):=h_{n-1}^{(\beta)}(x) h_{n-2}^{(\beta)}(x) \cdots h_{i}^{(\beta)}(x)
$$

and

$$
\widetilde{A}_{i}^{(\beta)}(x):=h_{i}^{(\beta)}(x) h_{i+1}^{(\beta)}(x) \cdots h_{n-1}^{(\beta)}(x)
$$

for integers $1 \leqslant i<n$ and $x \in R$. Then consider the finite product

$$
\mathfrak{G}:=A_{1}^{(\beta)}\left(x_{1}\right) A_{2}^{(\beta)}\left(x_{2}\right) \cdots A_{n-1}^{(\beta)}\left(x_{n-1}\right)=\sum_{w \in S_{n}} \mathfrak{G}_{w} \cdot \pi_{w} \in \operatorname{IdCox}_{\beta} .
$$

Next let $\widehat{\mathfrak{G}}:=m_{1} \mathfrak{G}$ and $\widehat{\mathfrak{G}}^{\mathrm{FPF}}:=m_{1}^{\mathrm{FPF}} \mathfrak{G}$. It is evident from $(9)$ that

$$
\widehat{\mathfrak{G}}=\sum_{z \in \mathcal{I}_{n}} \widehat{\mathfrak{G}}_{z} \cdot m_{z} \in \text { InvolMod }_{\beta} \quad \text { and } \quad \widehat{\mathfrak{G}}^{\mathrm{FPF}}=\sum_{z \in \mathcal{I}_{n}^{\mathrm{FPF}}} \widehat{\mathfrak{G}}_{z} \cdot m_{z} \in \text { FixedMod }_{\beta} .
$$

Proposition 3.2 is inefficient for computing $\widehat{\mathfrak{G}}_{z}$ since while $\mathfrak{G}$ contains $\left(\begin{array}{l}n \\ 2\end{array}\right)$ factors $h_{i}^{(\beta)}\left(x_{i}\right)$, it turns out that any $m_{z}$ can be written in the form $m_{z} \pi_{a_{1}} \pi_{a_{2}} \cdots \pi_{a_{p}}$ where $p \leqslant\left(\begin{array}{c}n_{1} \\ 2\end{array}\right)+\left(\begin{array}{c}n_{2} \\ 2\end{array}\right)$ for $n_{1}=\left\lceil\frac{n+1}{2}\right\rceil$ and $n_{2}=\left\lfloor\frac{n+1}{2}\right\rfloor$. We can derive an involution version of Proposition 3.2, however.

Lemma 4.3. For any integer $1 \leqslant i<n$ and elements $x_{i}, \ldots, x_{n-1}, y \in R$ it holds that

$$
\widetilde{A}_{i}^{(\beta)}(y) A_{i}^{(\beta)}\left(x_{i}\right) A_{i+1}^{(\beta)}\left(x_{i+1}\right) \cdots A_{n-1}^{(\beta)}\left(x_{n-1}\right)=\prod_{j=i+1}^{n-1} A_{j}^{(\beta)}\left(x_{j-1}\right) \cdot \prod_{j=i}^{n-1} h_{j}^{(\beta)}\left(x_{j} \oplus y\right) .
$$

Proof. Repeat the proof of $\left[6\right.$, Lem. 4.1] with the symbols $A_{i}, \widetilde{A}_{j}, h_{k}$ replaced by $A_{i}^{(\beta)}, \widetilde{A}_{j}^{(\beta)}, h_{k}^{(\beta)}$, and then apply the algebra anti-automorphism of $\operatorname{IdCox}_{\beta}$ that maps $\pi_{w} \mapsto \pi_{w^{-1}}$ to both sides.

For $i>j>0$, define $x_{i \oplus j}=x_{j \oplus i}:=x_{i} \oplus x_{j}=x_{i}+x_{j}+\beta x_{i} x_{j}$ and $x_{j \oplus j}:=x_{j}$.

Proposition 4.4. The following identities hold:

(1) We have $\widehat{\mathfrak{G}}=\prod_{i=1}^{n-1} \prod_{j=\min (i, n-i)}^{1} h_{i+j-1}^{(\beta)}\left(x_{i \oplus j}\right)$.

(2) If $n$ is even then $\widehat{\mathfrak{G}}^{\mathrm{FPF}}=\prod_{i=2}^{n-1} \prod_{j=\min (i-1, n-i)}^{1} h_{i+j-1}^{(\beta)}\left(x_{i \oplus j}\right)$.

In part (2), the indices $i$ and $j$ always satisfy $i>j>0$ so $x_{i \oplus j}=x_{i} \oplus x_{j}$.

Proof. We first prove part (1). The result is trivial when $n=1$ so assume $n \geqslant 2$. For any $1 \leqslant i<n$ we have $m_{1} \pi_{i} \pi_{i+1}=m_{s_{i+1} s_{i} s_{i+1}}=m_{1} \pi_{i+1} \pi_{i}$ and consequently $m_{1} h_{i}^{(\beta)}(x) h_{j}^{(\beta)}(y)=m_{1} h_{j}^{(\beta)}(y) h_{i}^{(\beta)}(x)$ for all integers $i, j$ and $x, y \in R$. Using this, one checks that $m_{1} A_{1}^{(\beta)}(x)=m_{1} \widetilde{A}_{1}^{(\beta)}(x)$, whence

$$
\begin{aligned}
\widehat{\mathfrak{G}} & =m_{1} A_{1}^{(\beta)}\left(x_{1}\right) A_{2}^{(\beta)}\left(x_{2}\right) \cdots A_{n-1}^{(\beta)}\left(x_{n-1}\right)=m_{1} \widetilde{A}_{1}^{(\beta)}\left(x_{1}\right) A_{2}^{(\beta)}\left(x_{2}\right) \cdots A_{n-1}^{(\beta)}\left(x_{n-1}\right) \\
& =m_{1} h_{1}^{(\beta)}\left(x_{1}\right) \widetilde{A}_{2}^{(\beta)}\left(x_{1}\right) A_{2}^{(\beta)}\left(x_{2}\right) \cdots A_{n-1}^{(\beta)}\left(x_{n-1}\right) .
\end{aligned}
$$


Applying Lemma 4.3 with $i=2$ and commuting $h_{1}^{(\beta)}\left(x_{1}\right)$ to the right gives

$$
\widehat{\mathfrak{G}}=m_{1} A_{3}^{(\beta)}\left(x_{2}\right) A_{4}^{(\beta)}\left(x_{3}\right) \cdots A_{n-1}^{(\beta)}\left(x_{n-2}\right) h_{1}^{(\beta)}\left(x_{1 \oplus 1}\right) h_{2}^{(\beta)}\left(x_{1 \oplus 2}\right) \cdots h_{n-1}^{(\beta)}\left(x_{1 \oplus(n-1)}\right) .
$$

We may assume by induction that

$$
\begin{aligned}
m_{1} A_{3}^{(\beta)}\left(x_{2}\right) A_{4}^{(\beta)}\left(x_{3}\right) \cdots A_{n-1}^{(\beta)}\left(x_{n-2}\right) & =m_{1} \prod_{i=1}^{n-3} \prod_{j=\min (i, n-2-i)}^{1} h_{i+j+1}^{(\beta)}\left(x_{(i+1) \oplus(j+1)}\right) \\
& =m_{1} \prod_{i=2}^{n-2} \prod_{j=\min (i, n-i)}^{2} h_{i+j-1}^{(\beta)}\left(x_{i \oplus j}\right) .
\end{aligned}
$$

This gives $\widehat{\mathfrak{G}}=m_{1} \prod_{i=2}^{n-2} \prod_{j=\min (i, n-i)}^{2} h_{i+j-1}^{(\beta)}\left(x_{i \oplus j}\right) \cdot \prod_{k=1}^{n-1} h_{k}^{(\beta)}\left(x_{1 \oplus i}\right)$, and it is not hard to see that this formula can be transformed by appropriate commutations to the expression in part (1). For instance, if $n=8$ then what needs to be shown is equivalent to the claim that one can turn the reduced word $3 \cdot 54 \cdot 765 \cdot 76 \cdot 7 \cdot 1234567$ into $1 \cdot 32 \cdot 543 \cdot 7654 \cdot 765 \cdot 76 \cdot 7$ using only relations of the form $i j \leftrightarrow j i$ for $|i-j|>1$.

The proof of part (2) is similar. Assume $n$ is even and $1 \leqslant i<n$. If $i$ is odd then $m_{1}^{\mathrm{FPF}} \pi_{i}=0$ and $m_{1}^{\mathrm{FPF}} h_{i}^{(\beta)}(x)=m_{1}^{\mathrm{FPF}}$ for all $x \in R$. On the other hand, if $i$ is even and $x, y \in R$ then

$$
m_{1}^{\mathrm{FPF}} \pi_{i} \pi_{i+1}=m_{1}^{\mathrm{FPF}} \pi_{i} \pi_{i-1} \quad \text { and } \quad m_{1}^{\mathrm{FPF}} h_{i}^{(\beta)}(x) h_{i+1}^{(\beta)}(y)=m_{1}^{\mathrm{FPF}} h_{i}^{(\beta)}(x) h_{i-1}^{(\beta)}(y) .
$$

Using these relations repeatedly we deduce that $m_{1}^{\mathrm{FPF}} A_{i}^{(\beta)}(x)=m_{1}^{\mathrm{FPF}} \widetilde{A}_{i+1}^{(\beta)}(x)$ for any odd integer $1 \leqslant i<n$. By Lemma 4.3, we therefore have

$$
\begin{aligned}
& \widehat{\mathfrak{G}}^{\mathrm{FPF}}=m_{1}^{\mathrm{FPF}} A_{1}\left(x_{1}\right) A_{2}\left(x_{2}\right) \cdots A_{n-1}\left(x_{n-1}\right)=m_{1}^{\mathrm{FPF}} \widetilde{A}_{2}^{(\beta)}\left(x_{1}\right) A_{2}^{(\beta)}\left(x_{2}\right) \cdots A_{n-1}^{(\beta)}\left(x_{n-1}\right) \\
& =m_{1}^{\mathrm{FPF}} A_{3}^{(\beta)}\left(x_{2}\right) A_{4}^{(\beta)}\left(x_{3}\right) \cdots A_{n-1}^{(\beta)}\left(x_{n-2}\right) \\
& \text { - } h_{2}^{(\beta)}\left(x_{1} \oplus x_{2}\right) h_{3}^{(\beta)}\left(x_{1} \oplus x_{3}\right) \cdots h_{n-1}^{(\beta)}\left(x_{1} \oplus x_{n-1}\right) .
\end{aligned}
$$

From here, the result follows by induction as in the proof of part (1).

Let $\triangle_{n}:=\{(i, j) \in \mathbb{Z} \times \mathbb{Z}: i \geqslant j>0\}$ and $\triangle_{n}^{\neq}:=\{(i, j) \in \mathbb{Z} \times \mathbb{Z}: i>j>0\}$. Equip these sets with the total order defined by $(i, j) \prec(k, l)$ if $i<k$ or if $i=k$ and $j>l$. An involution Hecke pipe dream for $z \in \mathcal{I}_{n}$ (respectively, $z \in \mathcal{I}_{n}^{\mathrm{FPF}}$ ) is a finite subset $D$ of $\triangle_{n}$ (respectively, $\triangle_{n}^{\neq}$) such that the word formed by listing the numbers $i+j-1$ as $(i, j)$ runs over $D$ in the order $\prec$ belongs to $\operatorname{InvHecke}(z)$. We write $\operatorname{InvDreams}(z)$ for the set of these subsets.

THEOREM 4.5. If $z \in \mathcal{I}_{n}$ or if $n$ is even and $z \in \mathcal{I}_{n}^{\mathrm{FPF}}$ then

$$
\widehat{\mathfrak{G}}_{z}=\sum_{D \in \operatorname{lnvDreams}(z)} \beta^{|D|-\widehat{\ell}(z)} \prod_{(i, j) \in D} x_{i \oplus j}
$$

where we set $x_{i \oplus i}:=x_{i}$ for $i>0$ and $x_{i \oplus j}:=x_{i}+x_{j}+\beta x_{i} x_{j}$ for $i>j>0$.

When $\beta=0$ our result reduces to $[8$, Thm. 1.5], which was proved in a different way using somewhat involved recurrences. The methods here give a new and simpler proof. For generic $\beta$, Theorem 4.5 resolves the symplectic half of [8, Problem 6.9].

Proof. First assume $z \in \mathcal{I}_{n}$. Part (1) of Proposition 4.4 implies

$\widehat{\mathfrak{G}}_{z}=\sum_{a=a_{1} \cdots a_{N} \in \operatorname{InvHecke}(z)} \beta^{N-\widehat{\ell}(z)} \sum_{\substack{0<\mathbf{i}=\left(i_{1} \leqslant \cdots \leqslant i_{N}\right) \in \operatorname{Compatible}(a) \\ i_{j} \leqslant a_{j}<2 i_{j} \forall j}} x_{i_{1} \oplus\left(a_{1}-i_{1}+1\right)} \cdots x_{i_{N} \oplus\left(a_{N}-i_{N}+1\right)}$.

One now checks that the map sending $(a, \mathbf{i})$ to $D=\left\{\left(i_{j}, a_{j}-i_{j}+1\right): 1 \leqslant j \leqslant\right.$ $\ell(a)\}$ is a bijection from the pairs indexing this double summation to the elements 
of $\operatorname{InvDreams}(z)$. When $n$ is even and $z \in \mathcal{I}_{n}^{\mathrm{FPF}}$, the same argument using part (2) of Proposition 4.4 gives the desired formula.

EXAMPLE 4.6. Suppose $n=4$. If $y=s_{3} s_{2} s_{3}=s_{2} s_{3} s_{2}=(2,4) \in \mathcal{I}_{n}$ as in Example 4.1, then the elements of InvDreams $(y)$ are the sets of nonzero positions in the matrices

$$
\left[\begin{array}{lll}
0 & 0 & 0 \\
1 & 1 & 0 \\
0 & 0 & 0
\end{array}\right], \quad\left[\begin{array}{lll}
0 & 0 & 0 \\
1 & 0 & 0 \\
1 & 0 & 0
\end{array}\right], \quad\left[\begin{array}{lll}
0 & 0 & 0 \\
1 & 1 & 0 \\
1 & 0 & 0
\end{array}\right],
$$

which are $\{(2,1),(2,2)\},\{(2,1),(3,1)\}$, and $\{(2,1),(2,2),(3,1)\}$. By Theorem 4.5,

$$
\widehat{\mathfrak{G}}_{y}=\left(x_{2} \oplus x_{1}\right) x_{2}+\left(x_{2} \oplus x_{1}\right)\left(x_{3} \oplus x_{1}\right)+\beta\left(x_{2} \oplus x_{1}\right) x_{2}\left(x_{3} \oplus x_{1}\right) .
$$

If $z=s_{3} \cdot s_{2} \cdot 1^{\mathrm{FPF}} \cdot s_{2} \cdot s_{3} \in \mathcal{I}_{n}^{\mathrm{FPF}}$ as in Example 4.1, then InvDreams $(z)$ contains just one element $\{(2,1),(3,1)\}$, and Theorem 4.5 asserts $\widehat{\mathfrak{G}}_{z}=\left(x_{2} \oplus x_{1}\right)\left(x_{3} \oplus x_{1}\right)$.

Acknowledgements. We thank Sergey Fomin for suggesting the problem of finding analogues of Macdonald's formulas for Schubert polynomials outside type A.

\section{REFERENCES}

[1] Sara C. Billey and Mark Haiman, Schubert polynomials for the classical groups, J. Amer. Math. Soc. 8 (1995), no. 2, 443-482.

[2] Sara C. Billey, Alexander E. Holroyd, and Benjamin J. Young, A bijective proof of Macdonald's reduced word formula, Algebr. Comb. 2 (2019), no. 2, 217-248.

[3] Sara C. Billey, William Jockusch, and Richard P. Stanley, Some combinatorial properties of Schubert polynomials, J. Algebraic Combin. 2 (1993), no. 4, 345-374.

[4] Anders S. Buch, A Littlewood-Richardson rule for the K-theory of Grassmannians, Acta Math. 189 (2002), no. 1, 37-78.

[5] Sergey Fomin and Anatol N. Kirillov, Reduced words and plane partitions, J. Algebraic Combin. 6 (1997), no. 4, 311-319.

[6] Sergey Fomin and Richard P. Stanley, Schubert polynomials and the nil-Coxeter algebra, Adv. Math. 103 (1994), no. 2, 196-207.

[7] Zachary Hamaker, Eric Marberg, and Brendan Pawlowski, Involution words: counting problems and connections to Schubert calculus for symmetric orbit closures, J. Combin. Theory Ser. A 160 (2018), 217-260.

[8] - Involution pipe dreams, https://arxiv.org/abs/1911.12009, 2019.

[9] Anatol N. Kirillov and Hiroshi Naruse, Construction of double Grothendieck polynomials of classical types using idCoxeter algebras, Tokyo J. Math. 39 (2017), no. 3, 695-728.

[10] Thomas Lam, Seung Jin Lee, and Mark Shimozono, Back stable Schubert calculus, https: //arxiv.org/abs/1806.11233, 2018.

[11] Ian G. Macdonald, Notes on Schubert polynomials, Laboratoire de combinatoire et d'informatique mathématique (LACIM), Université du Québec à Montréal, 1991.

[12] Eric Marberg, A symplectic refinement of shifted Hecke insertion, J. Combin. Theory Ser. A 173 (2020), Paper no. 105216 (50 pages).

[13] Eric Marberg and Brendan Pawlowski, K-theory formulas for orthogonal and symplectic orbit closures, Adv. Math. 372 (2020), Paper no. 107299 (43 pages).

[14] _ On some properties of symplectic Grothendieck polynomials, J. Pure Appl. Algebra 225 (2021), no. 1, Paper no. 106463 (22 pages).

[15] Brendan Pawlowski, Universal graph Schubert varieties, https://arxiv.org/abs/1902.09168, 2019.

[16] Benjamin Young, A Markov growth process for Macdonald's distribution on reduced words, https://arxiv.org/abs/1409.7714, 2014.

Eric Marberg, Hong Kong University of Science and Technology, Department of Mathematics, Clear Water Bay, Hong Kong

E-mail : eric.marberg@gmail.com

Brendan Pawlowski, University of Southern California, Los Angeles, California, USA

E-mail : br.pawlowski@gmail.com 